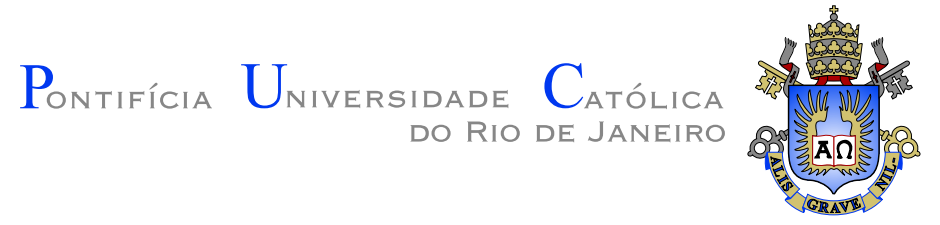

André Luiz dos Santos Lima

\title{
Avaliação de Técnicas de Projeto de Lentes Metálicas
}

Dissertação apresentada como requisito parcial para obtenção do grau de Mestre pelo Programa de Pós-graduação em Engenharia Elétrica do Departamento de Engenharia Elétrica da PUC-Rio

Orientador: Prof. José Ricardo Bergmann 


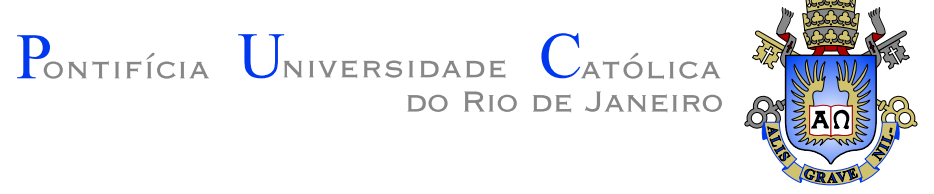

André Luiz dos Santos Lima

\title{
Avaliação de Técnicas de Projeto de Lentes \\ Metálicas
}

Dissertação apresentada como requisito parcial para obtenção do grau de Mestre pelo Programa de Pós-graduação em Engenharia Elétrica do Departamento de Engenharia Elétrica do Centro Técnico Científico da PUC-Rio. Aprovada pela comissão examinadora abaixo assinada.

\author{
Prof. José Ricardo Bergmann \\ Orientador \\ Departamento de Engenharia Elétrica - PUC-Rio
}

Prof. Flávio José Vieira Hasselman

Centro de Estudos em Telecomunicações - PUC-Rio

Prof. Sandro Rogério Zang

Centro de Estudos em Telecomunicações - PUC-Rio

Prof. Antônio Romero Sapienza

UERJ

Prof. José Eugênio Leal

Coordenador do Centro Técnico Científico - PUC-Rio

Rio de Janeiro, 20 de Dezembro de 2011 
Todos os direitos reservados. Proibida a reprodução total ou parcial do trabalho sem autorização da universidade, do autor e do orientador.

\section{André Luiz dos Santos Lima}

Graduou-se em Engenharia na PUC-Rio, cursando Telecomunicações e Eletrônica. Iniciou-se em pesquisa durante a graduação, trabalhando com acopladores coaxiais no laboratório de métodos computacionais em eletromagnetismo aplicado.

Ficha Catalográfica

Lima, André Luiz dos Santos

Avaliação de Técnicas de Projeto de Lentes Metálicas / André Luiz dos Santos Lima; orientador: José Ricardo Bergmann. - Rio de Janeiro : PUC-Rio, Departamento de Engenharia Elétrica, 2011.

v., 109 f: il. ; $29,7 \mathrm{~cm}$

1. Dissertação (Mestrado em Engenharia Elétrica ) - Pontifícia Universidade Católica do Rio de Janeiro, Departamento de Engenharia Elétrica.

Inclui referências bibliográficas.

1. Engenharia Elétrica - Tese. 2. Lentes Metálicas. 3. Lentes Dielétricas. 4. Síntese ótica. 5. Antenas. I. Bergmann, José Ricardo. II. Pontifícia Universidade Católica do Rio de Janeiro. Departamento de Engenharia Elétrica. III. Título. 
Aos meus pais. 


\section{Agradecimentos}

Ao meu orientador Professor José Ricardo Bergmann pelo apoio, paciência de sempre, e incentivo para a realização deste trabalho.

Ao CNPq e à PUC-Rio, pelos auxílios concedidos, sem os quais este trabalho não poderia ter sido realizado.

Ao pessoal do PAA, demais professores e funcionários pelos ensinamentos de todos os dias.

À Maria Cristina, pelo apoio, carinho, e, principalmente pela paciência. 


\section{Resumo}

Lima, André Luiz dos Santos; Bergmann, José Ricardo. Avaliação de Técnicas de Projeto de Lentes Metálicas. Rio de Janeiro, 2011. 109p. Dissertação de Mestrado - Departamento de Engenharia Elétrica, Pontifícia Universidade Católica do Rio de Janeiro.

Uma estrutura de placas condutoras apresenta características refrativas que permitem sua aplicação no projeto de lentes na faixa de microondas. Consiste de um conjunto de placas metálicas justapostas, paralelas entre si, estando estas afastadas umas das outras de uma distância maior que meio comprimento de onda e menor que um comprimento de onda, de forma que o campo elétrico da onda incidente esteja orientado paralelo as placas. As propriedades refrativas surgem do aumento da velocidade de fase da onda eletromagnética devido à presença das placas metálicas. A forma destas placas planas define a direção da onda que se propaga entre elas e permite o controle da distribuição de campo na interface de saída das lentes metálicas. O controle da distribuição amplitude e fase do campo na abertura destas lentes permite, consequentemente, o controle do diagrama de radiação do dispositivo. Neste trabalho, são utilizadas técnicas aproximadas de síntese e análise de lentes metálicas que são projetadas para maximizar o ganho do dispositivo. A técnica de síntese é baseada nas aproximações da ótica geométrica e a de análise combina estas aproximações com uma análise difrativa na interface destas lentes. Para avaliar a efetividade destes algorítimos de síntese e análise aproximadas, os dispositivos projetados são submetidos a uma técnica de análise rigorosa no domínio do tempo. São apresentados e discutidos os projetos de lentes metálicas iluminadas por cornetas piramidais e lentes corretoras para cornetas setoriais no plano $\mathrm{H}$.

\section{Palavras-chave}

Lentes Metálicas; Lentes Dielétricas; Síntese ótica; Antenas. 


\section{Abstract}

Lima, André Luiz dos Santos; Bergmann, José Ricardo (advisor). Evaluation of Techniques for the Design of Metallic Lenses. Rio de Janeiro, 2011. 109p. MSc. dissertation - Departamento de Engenharia Elétrica, Pontifícia Universidade Católica do Rio de Janeiro.

Metal plates structures exhibit refractive properties allowing the design of lens at microwave applications. A metal lens consist of a set of metallic plates placed side by side, parallel to each other, and away by a distance greater than half wavelength and less than a wavelength, so that electric field of incident wave is aligned parallel to the plates. Refractive properties arise from phase velocity increase of electromagnetic wave due to metal plate presence. The plate shape defines the wave propagation direction between the plates, allowing field distribution handling at lens output interface. Field distribution handle in aperture enable us, consequently, to control radiation pattern. In this work, an approximate techniques of synthesis and analysis for metal plates lens are outlined with the objective of improve structure gain. Geometric optics approach was used for synthesis technique, while, in analysis, diffraction effects was taken into account at surface lens. In order to evaluate the synthesis and analysis techniques, designed structures are submitted to a rigorous analysis in the time domain. Results for corrective lenses with $\mathrm{H}$ plane horns and metal plates lens in front of pyramidal horns are presented and discussed.

\section{Keywords}

Metallic Lenses ; Dielectric Lenses ; Optical Synthesis ; Antennas. 


\section{Sumário}

1 Introdução $\quad 13$

1.1 Motivação 16

$\begin{array}{lll}1.2 & \text { Estrutura do Trabalho } & 17\end{array}$

2 Lentes Metálicas $\quad 19$

2.1 Metal-Plate 19

2.2 Lentes 24

3 Teoria de Placas Metálicas Paralelas 30

3.1 Placas Paralelas 30

3.2 Natureza e Direção dos Campos Refletidos e Transmitidos 31

3.3 Amplitude e Fase do Campo Refletido e Transmitido 39

4 Cornetas Setoriais com Lentes $\quad \mathbf{5 2}$

4.1 Campos na Abertura das Cornetas Setoriais no plano H 53

4.2 Síntese de Lentes Metálicas 54

4.3 Projeto de Cornetas com Lente 58

4.4 Aproximação para o Campo Radiado pela Corneta com Lente 65 Aproximação para o Campo Radiado pela Corneta com Lente 65

4.5 Análise de Cornetas com Lentes 67

5 Cornetas Piramidais com Lentes $\quad \mathbf{7 9}$

5.1 Campos na Abertura das Cornetas Piramidais $\quad 81$

5.2 Sintese de Lentes para Cornetas Piramidais 82

5.3 Projeto de Lentes para Cornetas Piramidais 86

5.4 Aproximação para o Campo Radiado pela Lente Metálica 88

5.5 Análise de Lentes para Cornetas Piramidais 90

6 Conclusões 106

$\begin{array}{ll}\text { Referências Bibliográficas } & 108\end{array}$ 


\section{Lista de figuras}

2.1 Geometria das placas paralelas (Vista Superior). 19

2.2 Geometria das Placas Paralelas (Vista em Perspectiva). 20

2.3 Variação do índice de refração $\mathrm{n}$ com a distância entre placas dimensionada para propagação do modo $T E_{10}$. 22

2.4 Traçado de raios sobre a lente [6]. 23

2.5 Variação do índice de refração efetivo com a inclinação da onda incidente. 24

2.6 Perfil da lente dielétrica e geometria associada. 25

2.7 Perfil da lente metálica e geometria associada. 27

2.8 Lente E-plane. 28

2.9 Lente Côncava. 28

2.10 Lente Plana. 28

3.1 Geometria do Conjunto de Placas Paralelas. 31

3.2 Geometria para Reflexão. 37

3.3 Geometria para difracão. 38

3.4 Variação do ângulo da onda difratada. $\quad 39$

3.5 Diagrama de pólos e zeros. 44

3.6 Variação do Coeficiente de Transmissão com o Ângulo de Incidência. 49

3.7 Deslocamento de fase na Transmissão. 50

3.8 Variação da ondas refletidas e ângulo de reflexão para $n=0,4 . \quad 50$

3.9 Deslocamento de fase das ondas refletidas para $n=0,4$. 51

4.1 Geometria da Corneta Setorial Plano H. 52

4.2 Vista no Plano $\mathrm{H}$. 53

4.3 Geometria para Lente Plana. 55

4.4 Traçado de Raios Lente Plana. 56

4.5 Geometria para Lente Côncava.

4.6 Traçado de Raios Lente Côncava. 58

4.7 Diagrama de Radiação da Corneta - Exemplo I. 60

4.8 Distribuição de Campo no Interior da Corneta. 60

4.9 Corneta Setorial com Lente Plana. 61

4.10 Corneta Setorial com Lente Plana(Corte). 61

4.11 Diagrama de Radiação da Corneta - Exemplo II. 63

4.12 Corneta Setorial com Lente Côncava. 64

4.13 Corneta Setorial com Lente Côncava(Corte). 64

4.14 Geometria Frontal. 66

4.15 Diagrama de Radiação Corneta com Lente Plana Plano H - Caso Ideal. $\quad 69$

4.16 Diagrama de Radiação Corneta com Lente Plana Plano E - Caso Ideal 69

4.17 Diagrama de Radiação Corneta com Lente Plana Plano H - Deslocamento de Fase I. $\quad 70$ 
4.18 Diagrama de Radiação Corneta com Lente Plana Plano H - Deslocamento de fase II.

4.19 Amplitude Relativa do Campos nas Aberturas. $\quad 72$

4.20 Distribuição de Campo no Interior da Corneta com Lente. $\quad 72$

4.21 Diagrama de Probes para Lente Plana. 74

4.22 Diagrama de Radiação Corneta com Lente Plana Plano H - Comparativo.

4.23 Diagrama de Radiação Corneta com Lente Côncava Plano H - Caso Ideal.

4.24 Diagrama de Radiação Corneta com Lente Côncava Plano E - Caso Ideal.

4.25 Diagrama de Radiação Corneta com Lente Côncava Plano H Deslocamento de Fase I.

4.26 Diagrama de Probes para Lente Côncava.

4.27 Diagrama de Radiação Corneta com Lente Plana Plano H - Comparativo.

5.1 Geometria da Corneta Piramidal. 79

5.2 Vista no Plano E. $\quad 80$

5.3 Vista no Plano H. 80

5.4 Diagrama da Corneta Piramidal no Plano E. 84

5.5 Diagrama da Corneta Piramidal a $45^{\circ}$. 84

5.6 Diagrama da Corneta Piramidal no Plano H. 85

5.7 Corneta Piramidal com Lente. $\quad 87$

5.8 Conservação de Energia no Tubo de Raios.

5.9 Geometria para Campo Distante. $\quad 89$

5.10 Diagrama de Radiação no Plano H - Caso A. $\quad 91$

5.11 Diagrama de Radiação no Plano E - Caso A. 91

5.12 Campo na Abertura - Caso A (eixo x). 93

5.13 Campo na Abertura - Caso A (eixo y). $\quad 93$

5.14 Fase na Abertura - Caso A (eixo x).

5.15 Fase na Abertura - Caso A (eixo y).

5.16 Diagrama de Probes no Plano H - Caso A. 95

5.17 Diagrama de Probes no Plano E - Caso A. 95

5.18 Diagrama de Radiação Corneta com Lente Uniforme - Plano H. 96

5.19 Diagrama de Radiação Corneta com Lente Uniforme - Plano E. 96

5.20 Perda de Retorno - Corneta com Lente Uniforme. 97

5.21 Diagrama de Radiação no Plano H - Caso B. 99

5.22 Diagrama de Radiação no Plano E - Caso B. 99

5.23 Diagrama de Probes no Plano H - Caso B. 100

5.24 Diagrama de Probes no Plano E - Caso B. 100

5.25 Campo na Abertura - Caso B (eixo x). 101

5.26 Campo na Abertura - Caso B (eixo y). 101

5.27 Fase na Abertura - Caso B (eixo x). 102

5.28 Fase na Abertura - Caso B (eixo y). 102

5.29 Diagrama de Radiação Corneta com Lente Não Uniforme - Plano H.104

5.30 Diagrama de Radiação Corneta com Lente Não Uniforme - Plano E. 104

5.31 Perda de Retorno - Corneta com Lente Não Uniforme. 105 


\section{Lista de tabelas}

3.1 Coeficientes de Reflexão, Transmissão e Deslocamento de Fase. 48

4.1 Espaçamento $d$ e Índice de Refração $n$ para Lente Plana. 62

4.2 Comprimento das Placas para Lente Côncava. 63

4.3 Parâmetros da Corneta com Lente Plana. 68

4.4 Coordenada das Placas para Lente Plana. 68

4.5 Amplitude Normalizada e Fase do Campo Elétrico nas Aberturas. 71

4.6 Características do Diagrama de Radiação - Lente Plana. 73

4.7 Parâmetros da Corneta com Lente Côncava. 75

4.8 Características do Diagrama de Radiação - Lente Côncava. 78

5.1 Parâmetros da Corneta Piramidal. 83

5.2 Espaçamento $d$ e Índice de Refração $n$ para Lente. 87 
Faraway, so close Up with the static and the radio With satelite television You can go anywhere

U2, Zooropa, 1993. 